\title{
Mapeo cognitivo y exploración háptica para comprender la disposición del espacio de videntes e invidentes'
}

Luis B. Sanabria R.

Artículo recibido: 11-8-2006 y aprobado: 2-5-2007

\section{Cognitive mapping and haptic exploration to understand} blind and sight people space configuration

Resumen: La forma como videntes e invidentes representan el espacio genera una fuente de conocimiento que nos permite estudiar nuevas alternativas para orientar procesos de aprendizaje en la movilidad y orientación de personas que carecen de visión. Este documento caracteriza el proceso de razonamiento espacial a partir del conocimiento del mapeo cognitivo y plantea una serie de antecedentes de investigación que nos llevan a comprender la representación espacial de las personas. El mapeo cognitivo, como un proceso de razonamiento espacial, nos indica cómo se movilizan los videntes e invidentes y qué información se requiere en la movilidad, lo mismo que la manera como está distribuida dicha información en el ambiente. En los invidentes estas tareas requieren del estudio de procesos de exploración háptica que utilizan órganos sensoriales paralelos -visión alternativa-para identificar la representación del ambiente. Experiencias en la formación de modelos mentales espaciales como las referidas en este escrito, señalan que la construcción de mapas cognitivos, tanto de videntes como invidentes, es una estrategia poderosa que suministra información espacial para la movilidad. Este factor interviene en el mejoramiento de las habilidades de orientación y búsqueda del camino, lo cual influye en la orientación, independencia y calidad de vida de las personas invidentes (Jacobson, 1998).

Palabras clave: mapeo cognitivo, representación espacial, exploración háptica, movilidad.
Abstract: The way in which sighted and blind people represent themselves in the space, creates a knowledge source that allows us to study new alternatives to guide learning processes in the mobility and orientation in blind people. This article characterizes the spatial reasoning process from the knowledge of the cognitive mapping and also considers a set of research background that lets us to understand people' spatial representation. The cognitive mapping as a reasoning spatial process shows us the way in which sighted and blind people move, the kind of information required in the mobilization, as well as the way this information is distributed in the environment. In blind people these tasks required the study of haptic exploration processes that use parallel sensorial organs -alternative vision- to identify the environment representation. Experiences in the spatial mental model formation as referred in this article show that the cognitive mapping creation not only in sighted but in blind people, is a powerful strategy that provides spatial information to mobility. This factor intervenes in orientation abilities and road search which influence in orientation, independence and blind people quality life (Jacobson, 1998).

Key words: cognitive mapping, spatial representation, haptic exploration, mobility.

1 Este documento hace parte del desarrollo de la tesis de doctorado del programa interinstitucional de Doctorado en Educación de la Universidad Pedagógica Nacional.

* Profesor de la Universidad Pedagógica Nacional. lubsan@pedagogica.edu.co 


\section{Introducción}

El espacio es fundamental para la existencia humana y tiene una gran influencia en el pensamiento humano (Jacobson, 1998). La gente vive e interactúa en un tiempo y espacio continuos; los individuos acceden y negocian el conocimiento espacial del mundo a través de sus sentidos. Acceder a la información espacial a través de mapas, diagramas, gráficos, es fundamental para la educación y la navegación.

Todo el proceso educativo y la visualización de la vida diaria son métodos importantes para comprender información compleja y navegar alrededor de información estructurada (Jacobson, 2002). La calidad de vida tanto en videntes como en invidentes depende en gran medida de su habilidad para inferir información de decisiones espaciales a través del procesamiento y síntesis de la información espacial de una variedad de situaciones a diferentes escalas (Golledge, 1993). Para limitar estas variantes, el ser humano tiene un conocimiento espacial del entorno que lo rodea.

El conocimiento de lugares en el mundo se da a través de una variedad de estimulación de nuestros sentidos. Estas entradas sensoriales emergen directamente del ambiente, donde los sentidos entran en percepción directa con los objetos cercanos. Los procesos cognitivos son generados a partir de la mediación entre las entradas sensoriales del ambiente y la información espacial. La experiencia sensorial a través de la contemplación se transforma en conocimiento y comprensión, y genera lo que se denomina mapeo cognitivo (Jacobson, 1998). El mapeo cognitivo, como un proceso de razonamiento espacial, indica cómo se movilizan los videntes e invidentes y qué información se requiere en la movilidad, lo mismo que la manera como está distribuida dicha información en el ambiente. Para estudiar estos procesos nos centramos en la exploración háptica como un medio para identificar la representación del ambiente a través del tacto.

Referentes al estudio de la representación espacial, los conceptos tratados en el marco de este documento plantean en primera instancia la comprensión de fenómenos que hacen parte del mapeo cognitivo. En segunda instancia, tratan los mecanismos de exploración háptica en la representación espacial. Finalmente, consideran la exploración del espacio a través de mapas táctiles.

\section{Mapeo cognitivo}

La habilidad para aprender una ruta y repasarla desde la memoria es un aspecto que corresponde al conocimiento del mapa cognitivo elaborado por las personas. Este conocimiento incluye todos los aspectos de codificación, procesamiento y recuperación de información acerca del ambiente (Blades et al., 2002; Golledge, 1999). Down y Stea (1973, p. 9) definen el mapeo cognitivo como "un proceso compuesto de una serie de transformaciones psicológicas por medio de las cuales un individuo adquiere, almacena, recupera y decodifica información acerca de localizaciones relativas y atributos del fenómeno en su ambiente espacial cotidiano".

En un amplio sentido, un mapa cognitivo puede ser pensado como un modelo interno del mundo en que vivimos (Golledge y Stimpson, 1997). Existen dos elementos que se unen para con- 
formar el mapa cognitivo: la cognición espacial y el conocimiento del mundo (Kitchin, 1994). El componente espacial concierne a la estructura, relaciones y entidades del espacio (Hart y Moore, 1973), mientras que el componente ambiental se relaciona con los atributos físicos que componen un lugar (Hart y Conn, 1991).

El conocimiento espacial está retenido en la mente como una clase de imaginería mental. La imaginería puede alcanzar desde una escena de un cuadro (un rostro que se recuerda, por ejemplo), hasta una escena compleja y artificial (un sueño) (Robinson et al., 1978). En la imaginería mental existe un alto grado de disposición espacial, donde están los conceptos que van desde lo simple, como cerca o lejos, a lo complejo e ideas bien desarrolladas, como la posición de objetos en un espacio geométrico conceptualizado.

El conocimiento de estas localizaciones y relaciones forma nuestras imágenes cognitivas del ambiente y lleva a cabo nuestro comportamiento y la composición de una decisión espacial (Jacobson, 1992). Las imágenes almacenadas en nuestro cerebro forman los mapas cognitivos o mapas mentales y la representación del espacio geográfico en un dibujo es lo que se conoce como mapa visual (Robinson et al., 1978). Un mapa visual puede tomar muchas formas, figuras, escalas y propósitos. Las disposiciones espaciales complicadas pueden estar mostrando la transferencia de ideas y conceptos.

Así como los mapas visuales son una herramienta poderosa para la comprensión y educación de personas videntes, los mapas táctiles cumplen la misma función en personas invidentes. Un mapa táctil está diseñado y construido para ser leído por el sentido del tacto. Para ello requiere que cada parte sea dibujada en alto relieve (Walter y Robinson, 1983). Para los ciegos, un mapa táctil es el único medio de adquirir un conocimiento estructurado para organizar espacios y lugares. Su mapa cognitivo difiere del de personas videntes debido a las diferentes entradas sensoriales que forman su imagen mental, lo cual hace difícil la fijación de una precisión de la imagen. Los mapas visuales que construye el ciego pueden ser considerados precisos a partir de otros criterios, diferentes a la precisión planimétrica. Estos criterios de precisión para el ciego pueden ser considerados por la utilidad, más que la completitud o precisión.

Jacobson (1992) plantea que en la conceptualización de lugares a pequeña escala los videntes e invidentes son capaces de percibir su ambiente cercano de manera simple con un mínimo uso de la memoria. Durante el mapeo cognitivo nosotros percibimos escalas locales y utilizamos grandes escalas cognitivas. Sin embargo, el límite escalar es muy difuso y difiere en videntes e invidentes. El umbral de área de los sentidos es diferente y el sentido de la visión tiene un mayor umbral de área que los sentidos restantes. Uno puede ver más lejos que lo que puede percibir y oler. El ciego está forzado a percibir grandes espacios de manera secuencial. Su imagen interna es de naturaleza conceptual y está comprometida a la memoria. Su ventana del mundo es más pequeña que para una persona que puede ver. Para ver un ambiente a gran escala, los 
videntes utilizan los mismos métodos de integración de imágenes que utiliza el ciego en ambientes pequeños.

Jacobson (1998) sugiere cuatro razones para investigar las habilidades de invidentes en la generación de mapas cognitivos:

1. El mapeo cognitivo provee señales de cómo mejorar las habilidades de orientación y búsqueda del camino que influyen en la orientación, movilidad, independencia y calidad de vida de las personas invidentes.

2. El mapeo cognitivo provee la base de conocimiento acerca de cómo los invidentes navegan, qué información se necesita en el proceso de navegación y cómo se presenta esta información (Kitchin et al., 1997).

3. El conocimiento y la comprensión del mapeo cognitivo podrían utilizarse en la planeación de ambientes que son fáciles de recordar.

4. El estudio de la cognición espacial de las personas con deficiencias sensoriales genera información valiosa, obtenida del rol de la experiencia sensorial en el mapeo cognitivo.

Kitchin y Jacobson (1997) plantean que el interés de entender cómo las personas invidentes comprenden el espacio, podría conducir a la planeación de ambientes fáciles de recordar y utilizar. De la misma forma, la comprensión del espacio podría generar conocimiento acerca del contenido, la forma y la localización de la información espacial, para que sea disponible a los peatones invidentes. Adicionalmente, la comprensión del espacio podría proveer señales acerca de cómo mejorar las habilidades de orientación y búsqueda del camino, proporcionando retroalimentación sobre conocimiento y estrategias de pensamiento. Esta retroalimentación podría proveer a los docentes de movilidad con información de cómo enseñar estrategias más efectivas de pensamiento, y a los diseñadores, de dispositivos con puntos de referencia para medir el impacto y efectividad de las estrategias de entrenamiento y ayudas de movilidad (Kitchin y Jacobson, 1997).

Con relación a la eficacia y eficiencia de dispositivos diseñados como ayudas para la orientación y navegación de invidentes, se hace necesario que su concepción y diseño se realicen con base en el conocimiento de cómo navegan los invidentes sin estas ayudas. La investigación en mapeo cognitivo puede proporcionar este conocimiento, sugiriendo la información necesaria para que el invidente pueda navegar y la forma como se debe presentar esta información (Kitchin et al., 1997). La investigación en mapeo cognitivo permite explorar cómo los invidentes aprenden, comprenden y piensan acerca del espacio geográfico (Passini y Proulx, 1988; Kitchin et al., 1997).

De acuerdo con las investigaciones desde la geografía, la psicología, la planeación urbana y la ciencia cognitiva, existen técnicas para acceder al conocimiento del mapeo cognitivo de videntes e invidentes. Una de estas técnicas es la basada en la ruta (Kitchin y Jacobson, 1997). En esta técnica, el razonamiento del aprendiz acerca del espacio tiene que ver con un conjunto de objetos conectados por caminos (Hunt y Waller, 1999): por ejemplo, Bogotá está localizada al final de la línea recta conocida como La Caro que se extiende desde Sopó hasta la capital. Bogotá está a 50 km de Sopó. 
Otras son las técnicas configuracionales (Kitchin y Jacobson,1997), en las que el razonamiento del aprendiz acerca del espacio es un sistema de posiciones donde se calcula la distancia y el sentido (Hunt y Waller, 1999); por ejemplo, la ciudad de Bogotá está localizada a $20^{\circ}$ de longitud este y $40^{\circ}$ de latitud norte.

El objetivo de las técnicas basadas en la ruta es determinar un conocimiento del individuo de la relación entre dos localidades y cómo desplazarse entre ellas. Las pruebas para medir este tipo de conocimiento se dividen en tres categorías (Kitchin y Jacobson, 1997):

- En la primera categoría, se cuestiona a los individuos acerca de la forma como repasan o infieren la ruta. Passini, Proulx y Rainville (1990) compararon la habilidad para seguir las huellas de una ruta desde el destino al punto de origen en videntes e invidentes. De la misma forma, Klatzky et al. (1990) compararon la habilidad de videntes e invidentes para retornar al punto de origen, después de que ellos atravesaron una ruta compuesta por dos y tres segmentos.

- En la segunda categoría, los sujetos pueden ser cuestionados con relación a estimar la distancia entre los nodos de partida y finalización de una ruta o los segmentos que constituyen esa ruta. Montello (1991) diseña pruebas de clasificación para medir la distancia cognitiva estimada. Para ello describe técnicas de escalado de radio, como la estimación de magnitud, donde se requiere que los sujetos asignen un número a una distancia a escala en relación con un valor dado. Por ejemplo, a la distancia entre dos ciudades se le podría asignar un valor a escala de 100. Este valor serviría para que el sujeto estime la distancia de otros lugares. Otra técnica es la estimación del radio, donde se requiere que el sujeto marque en una línea la distancia de un lugar. La longitud de la línea representa la distancia dada. Otras técnicas de escalado ordinal o de intervalos requieren que los individuos decidan sobre varias distancias para saber cuál es la más larga (Biel, 1982; Allen, 1981) y clasifiquen en orden de longitud (Allen, et al., 1978). De la misma forma, asignen una determinada distancia a intervalos que parezcan ser iguales (Cadwallader, 1979).

- En la tercera categoría, los individuos son cuestionados en función de estimar la dirección entre los nodos de inicio y fin correspondientes a una ruta o entre varios lugares a lo largo de la ruta. En este método se requiere que los individuos imaginen que ellos están parados en un sitio y el punto de destino en otro. Con esta posición, ellos dibujan una línea representando la dirección del punto de destino (Bigelow, 1991).

Las técnicas configuracionales incluyen cuatro categorías para medir el conocimiento configuracional (Kitchin y Jacobson, 1997):

1. Primera categoría. Se refiere a los métodos de prueba gráfica donde se incluyen todas las variaciones en la elaboración de bosquejos de mapas. En esta línea existen cuatro variaciones de mapeo básicas:

- La primera es la técnica de elaboración de mapas básicos que consiste en obtener del sujeto un dibujo libre que incluya un boceto mínimo del mapa 
solicitado por el investigador. La tarea consiste en darle al sujeto un papel en blanco para que éste dibuje un mapa de un ambiente determinado.

- La segunda es la técnica de mapeo normal. Esta técnica impone más restricciones al sujeto que la anterior. En esta tarea el investigador se interesa por características más específicas. Para ello, él podría formular instrucciones apropiadas para obtener los datos requeridos.

- La tercera técnica consiste en un mapeo de señales, donde el investigador le proporciona al sujeto una parte de un mapa y se le solicita que complete las características específicas.

- La cuarta técnica de mapeo longitudinal le permite estudiar al investigador la manera como va evolucionando la elaboración del mapa. Para ello, el sujeto elabora el mapa por partes en diferentes hojas sobrepuestas teniendo en cuenta ciertos intervalos de tiempo.

Jacobson (1992) utilizó el procedimiento de mapeo normal para reunir dibujos que representaban el conocimiento de individuos ciegos de un campus universitario. En este estudio, los sujetos usaron líneas realzadas para bosquejar el campo sobre una mesa de dibujo, luego de aprender un mapa táctil del área. Los sujetos fueron capaces de dibujar el campus con un nivel de precisión alto, comparado con el mapa táctil del área.

2. Segunda categoría. Consiste en los métodos de prueba de reconstrucción y gráfica parcial. Estos métodos proveen al individuo con cierta cantidad de información espacial asistida, donde las metodologías para responder a las señales espaciales son esencialmente verificadoras de localización. Estos métodos difieren de los métodos de prueba gráfica en que únicamente requieren de la localización de puntos. Esto reduce la habilidad motora para dibujar y provee a los individuos de un marco estructural de respuestas. Una de las técnicas utilizada por Thomdyke y Hayes-Roth (1982) consistía en pedir a los sujetos que ubicaran un lugar en relación con dos puntos: uno ubicado al iniciar una ruta, y otro localizado arbitrariamente. Este método provee una escala y una orientación al sujeto. Esta teoría utilizada en este estudio investigativo permite pensar en disponer un mapa háptico compuesto por agujeros para que los invidentes, una vez han sido entrenados en ubicación de objetos reales con un dispositivo mecatrónico, ubiquen los mismos objetos a escala en cada agujero de acuerdo con la memorización de ubicación de los mismos, respondiendo a la relación que existe entre el objeto que se va a ubicar y otros situados en el área de ubicación.

3. Tercera categoría. Son las técnicas unimultidimensionales que utilizan datos de conocimiento de ruta para explorar lo que está oculto o inferir la estructura de conocimiento configuracional. El proceso que siguen los sujetos en esta tarea consiste en construir un espacio bidimensional a partir de un dato unidimensional que proviene del uso de una serie de algoritmos. En esta categoría se distinguen dos técnicas:

- La técnica de escalado multidimensional está diseñada para construir un mapa mostrando las relaciones entre un número de objetos, pero únicamente dando una matriz de 
distancias entre ellos (Aitken et al., 1989). El propósito de esta técnica es descubrir el patrón o estructura a través de un conjunto de datos empíricos y representarlos visualmente (Golledge, 1977). Lockman, Rieser y Pick (1981) estudiaron la estimación de distancia euclidiana en videntes e invidentes. A los individuos se les presentaban los nombres de tres lugares para que determinaran los dos más lejanos y los dos más cercanos. La serie de respuestas de los sujetos fueron graficadas en escalas multidimensionales para generar mapas bidimensionales.

- La técnica de convergencia proyectiva, también llamada método de resección, utiliza la estimación de dirección para trabajar las coordenadas de los diferentes lugares. Los individuos estiman la distancia y la dirección a lugares inadvertidos a partir de tres o más lugares. Los vectores resultantes dibujados y los puntos donde finalizan las líneas forman un triángulo de error cuyo punto medio (cruce de las medianas) se considera como la localización cognitiva del sitio. Hardwick et al. (1976) desarrollaron esta técnica en un estudio, donde los sujetos primero se familiarizaban autónomamente con cuatro lugares ubicados en una librería. Después, los sujetos, utilizando un tubo, señalaban la dirección de los lugares vistos. La localización cognitiva se calculaba con el triángulo de error formado por la intersección de la prolongación de las líneas que seguían la dirección de cada tubo. Kirasic et al. (1984) replicaron el experimento, pero esta vez consideraron, además de la dirección, la distancia estimada por los sujetos. Los sujetos utilizaban las dos variables para dibujar las líneas del triángulo de error.

4. Cuarta categoría. Se relaciona con los métodos de prueba de reconocimiento. Estos reúnen los datos del conocimiento configuracional, proporcionándole a los individuos una representación de un ambiente para que identifiquen rasgos y configuraciones de manera correcta, como reconocer un mapa o una fotografía aérea. Evans y Pezdek (1980) y Kitchin (1995) experimentaron con sujetos a quienes se les daban configuraciones de lugares ubicados estratégicamente. Únicamente la mitad de las configuraciones que ellos observaban o palpaban eran correctas. Algunas configuraciones se rotaban a diferentes ángulos. Las configuraciones se le mostraban a los sujetos por grupos de tres. Una vez ellos observaban estas disposiciones, se les preguntaba para que respondieran qué lugares, con relación a otros, estaban localizados correctamente.

En general, las pruebas de reconocimiento tienen una gran utilidad para medir el conocimiento configuracional de individuos impedidos visualmente. Aunque estas pruebas no ofrecen una confiabilidad ilimitada, sí generan alternativas de estudio y abren un panorama en la investigación de la forma como los individuos representan el espacio. De la misma forma, estas pruebas podrían revelar implicaciones importantes con respecto a la planeación de estrategias y la educación en movilidad.

La movilidad es una actividad compleja que requiere la integración de la percepción y la cognición junto a las ha- 
bilidades perceptuales y representacionales (Warren y Kocon, 1974; Jacobson, 1998). Un ejemplo de este planteamiento se da con un individuo vidente que no puede ver el destino desde un punto de origen; sin embargo, él es capaz de usar su representación cognitiva para inferir la dirección y alimentar su mapa cognitivo (Garling et al., 1984). Esta representación hipotética del espacio no se puede observar directamente, es un conocimiento internalizado subjetivo del ambiente. Esta información no necesita tener una métrica; sin embargo, el entrenamiento y la experiencia podrían ayudar a estructurar la cognición. Para el caso de invidentes, el acceso al conocimiento de su mapa cognitivo requiere dos opciones (Kitchin y Jacobson, 1997): una es la externalización de un mapa cognitivo que se obtiene, se mide y se estudia de la misma forma como se estudia un mapa normal. Esta técnica es útil para inferir conocimiento y el comportamiento de los posibles resultados de pruebas. La otra es la acción de los sujetos para completar una tarea apropiada de su ambiente, como seguir una ruta o encontrar un camino. Esta tarea involucra a los participantes en la interacción con el espacio que se da con base en la exploración háptica que pone de manifiesto la acción de los sentidos restantes, como el tacto.

\section{Exploración háptica e integración de señales perceptivas}

La base de los invidentes para hacer juicios espaciales está en sus sentidos restantes. Palpar un mapa táctil es un método que ayuda a mejorar el conocimiento del espacio de las personas ciegas (Andrews, 1983). La háptica estudia e investiga cómo puede combinarse la modalidad sensorial del tacto con un mundo virtual generado por un computador. La información que se extrae mediante la háptica es intermodal, en cuanto integra, al menos, dos modalidades perceptivas: la propiocepción, que informa mediante cinestesias del estado del aparato esquelético-locomotor (tono muscular, posición del cuerpo, equilibrio, etc.) y la dermocepción o tacto que contribuye aportando información sobre la textura y la presión mecánica sobre la piel.

En contraste con la visión que es un sentido distal con una región foveal pequeña y una gran periferia de baja agudeza, el tacto es un sentido de contacto con múltiples regiones de agudeza alta (como comparar la superficie de los dedos donde cada dedo vendría a ser reemplazado por una fóvea) y periferia relativamente pequeña de baja agudeza (el alcance de las manos comparado con el alcance de los ojos es mucho más pequeño). Estas diferencias podrían hacer que el espacio explorado y anticipado pudiera ser confundido rápidamente en la representación mental del observador (Intraub, 2004). La noción psicológica de que "el tacto enseña a la visión" ha trascendido de tal manera que la entrada háptica puede ser utilizada para probar la confiabilidad de ciertas señales visuales (Atkins et al., 2001). La exploración háptica de una región delimitada de una escena sin la visión genera una representación más verídica de los límites del entorno y reduce los errores unidireccionales que caracterizan la extensión del límite. Este proceso involucra el tacto y el movimiento. La memoria, en este caso, se utiliza para el manejo de la extensión deliberada de 
los movimientos de la mano a través de pequeñas regiones delimitadas y para proveer información que podría prevenir errores con respecto a la expansión del espacio explorado (Intraub, 2004).

La visión y la háptica comparten el problema común de percibir una escena. Los dos sentidos deben proveer al perceptor con una coherente representación de un ambiente continuo de manera que pueda ser explorado una parte por una sola vez. O `Reagan (1992) ilustra a través de una analogía la similitud entre las exploraciones hápticas y visuales. Por ejemplo, si uno sostiene un bolígrafo en la mano sin estar viéndolo, uno percibe el bolígrafo aunque los receptores sensoriales provean información sobre la forma de la mano. La carencia de un conocimiento específico acerca del tipo de mina del bolígrafo no afecta al perceptor; análogamente, sucedería lo mismo con la carencia de un detalle de la periferia de baja agudeza de la visión. Si se desea conocer el tipo de mina, con un movimiento de la mano similar a un escaneo visual, se podría saber acerca de ese detalle. Esto lleva a concluir que la representación mental del ambiente no requiere una representación simultánea de todos los detalles para generar una representación útil; pero sí debe proveer una representación coherente que incorpore nueva información, cuando el perceptor desee conocer nuevas regiones. Esta visión se complementa con el planteamiento de Revesz (1950), quien sugirió que el reconocimiento háptico de objetos se da a partir de la percepción de partes del objeto, seguida por un proceso cognitivo a través del cual se integran dichas partes para configurar el objeto. La modalidad háptica provee al perceptor con una sucesión de visiones parciales de un mundo continuo, debido a que de alguna manera el resultado de la representación mental refleja la coherencia y continuidad del espacio circundante (Intraub, 2004).

La investigación de Intraub acerca de la extensión del límite avanza en la teoría de que uno de los medios por los cuales se logra esta coherencia es la proyección de una disposición anticipada de la escena. La región que se extiende en la representación mental del observador posee un potencial que refleja propiedades invariantes de escenas del mundo que representan la continuidad de la disposición, permitiéndole a éste comprender e integrar visiones sucesivas.

\section{Órganos sensoriales paralelos o visión alternativa}

Con relación a los órganos sensoriales paralelos, se prevé el uso de información táctil de los sensores de la piel para que las personas invidentes puedan representar el espacio. Este aspecto se incluye en la fase experimental del estudio desarrollado en la tesis doctoral, lo cual comparte la tesis de Millar (1997), quien plantea que todos los canales sensoriales (proveedores de información) convergen, aportando información alternativa a la proporcionada por la visión para la representación del espacio circundante.

De acuerdo con Brewster y Brown (2004), el sentido del tacto se divide aproximadamente en dos partes: cinestésico y cutáneo. El término cinestésico es utilizado para describir la información que surge de fuerzas y posiciones sensadas por los músculos y las articula- 
ciones. La percepción cutánea se refiere a los mecanorreceptores contenidos en la piel. El término cutáneo incluye las sensaciones de vibración, temperatura, dolor y sangrado ${ }^{2}$.

Millar (1997) y Rosa y Ochaíta (1993) sostienen que a través de la estimulación sensoriomotriz el ser humano se estructura en los ámbitos: cognitivo, afectivo y social. Una instancia que nos permite conceptualizar el proceso de representación del espacio es la localización táctil de puntos de contacto esparcidos en un escenario formado por mojones dispuestos a escala en un mapa háptico. Esta configuración del escenario ofrece una variedad de superficies u objetos tangibles que proveen información a una persona invidente para elaborar su representación mental del escenario, en el cual se va a desplazar con ayuda de un dispositivo háptico, extrapolando esta representación a la identificación de objetos reales que están ubicados en la misma forma que el mapa háptico.

En esta dimensión, la investigación en la tesis doctoral planea realizar un estudio de la percepción del invidente a partir de la exploración cinestésica o percepción de los movimientos musculares de los dedos y la sensibilidad de la piel que recibe información háptica de algún dispositivo. El proceso que se pensaría poner a prueba incluye tres etapas: la primera consiste en explorar un mapa háptico a escala de manera táctil, usando información percibida de manera cinestésica; la segunda consiste en realizar una réplica del mapa táctil para

2 La piel es el órgano más grande del cuerpo, mide aproximadamente $2 \mathrm{~m}^{2}$ (Montagu, 1971). La piel es un medio poderoso para comunicar información. ver su representación configuracional del espacio; y, la tercera se relaciona con la exploración de un ambiente real con un dispositivo que sensa información del medio y la transmite al cerebro por medio de la piel y el bastón. El uso de estos dos elementos nos permitiría ver el mapa cognitivo que construye la persona invidente. Esto hace suponer que la representación mental concebida en la exploración del mapa háptico provee suficiente información para extrapolar la configuración del espacio en un escenario real. De la misma forma, esta prueba conduciría a identificar el proceso que sigue el invidente para representar el espacio.

Klatzky y Lederman (2003) estudian la representación de la disposición y localización espacial a partir de puntos de contacto cinestésico de los dedos para determinar la habilidad de las personas en volver a los sitios que habían percibido con el tacto y reportar su disposición en el espacio. La finalidad de la experiencia consistió en determinar la naturaleza de la representación que se forman las personas de los puntos de contacto esparcidos en el escenario. Inicialmente, los dedos de los sujetos fueron guiados para localizar puntos en el espacio; luego los sujetos, sin guía, retrocedían ubicando sus dedos en los puntos aprendidos, hasta llegar a la posición inicial, reportando las propiedades métricas de la disposición espacial. Cuando la gente explora una región del espacio alcanzable por sus manos (espacio manipulatorio o peripersonal), retiene una variedad de superficies y objetos tangibles. Frecuentemente el contacto con uno o dos puntos de localización dispersos es suficiente para determinar 
la disposición de los objetos y guiar su alcance y manipulación.

Klatzky y Lederman (2003) concluyeron que las personas se forman una o más representaciones a partir de localizaciones táctiles, derivando respuestas a preguntas de puntos contactados espacialmente. Estas representaciones son operativas y dependen de las instrucciones de la tarea que se va a manejar. Para los autores una representación cinestésica parece retener información de localización, soportando datos precisos de localizaciones que han sido percibidas previamente a través del tacto.

Según Klatzky y Lederman (2003), se distinguen varias clases de representación para el espacio manipulatorio o peripersonal. Estas representaciones se dan en términos de un sistema de referencia y los parámetros que provee el mismo sistema: la primera se refiere a la representación cinestésica de localizaciones percibidas de manera háptica. Este tipo de representación egocéntrica provee un código en términos de entradas sensoriales de músculos, tendones y articulaciones, el cual le permite a los sujetos retornar posiciones que han palpado previamente en el espacio, sin comunicar directamente la distancia y ángulo entre éstas. La segunda es una representación espacial háptica extrínseca que se relaciona con las localizaciones del espacio externo. Una representación extrínseca puede ser egocéntrica o alocéntrica. Este tipo de representación utiliza un código espacial extrínseco que le permite a la gente retornar a posiciones contactadas previamente en el espacio, sin calcular directamente sus propiedades métricas. La tercera es una representación configuracional que localiza puntos salientes o mojones en una disposición relativa del espacio. Los ángulos indican la forma de la disposición, y las distancias, su escala. Este tipo de representación no provee directamente información necesaria para retornar a las localizaciones percibidas por el tacto.

Arno et al. (2001) investigaron el reconocimiento de patrones con videntes e invidentes. Para ello utilizaron un ambiente computacional donde se mostraban diferentes patrones formados por líneas. Los sujetos portaban un par de audífonos conectados al computador. Su trabajo consistía en mover un bolígrafo óptico sobre una tableta graficadora a medida que escuchaban sonidos que dependían de un código para orientar la dirección del movimiento de la mano. Este código identificaba los píxeles que formaban una línea recta, los cuales eran convertidos por el computador en sonidos armoniosos que se transmitían a los audífonos para que el sujeto identificara, a través de estos sonidos, la secuencia de puntos que él podía replicar con el bolígrafo o el cambio de dirección de esa secuencia de puntos de acuerdo con los cambios en el sonido que él recibía. La configuración de estas secuencias de puntos formaban diferentes figuras geométricas que establecían los patrones visuales en el computador. La acción del sujeto en la tableta graficadora le permitía revelar verbalmente el tipo de patrón que lograba describir con el bolígrafo.

Los resultados del experimento mostraron que tanto los videntes con los ojos vendados como los invidentes, quienes recibieron retroalimentación acústica en tiempo real, para relacio- 
narla con el movimiento de sus manos, reconocen los patrones visuales producidos por el computador. Arno et al. (2001) concluyeron que el reconocimiento de patrones visuales con el dispositivo utilizado en su experimento implicaba la identificación de las líneas orientadas en diferentes direcciones y las relaciones espaciales entre éstas, lo cual es consistente con los planteamientos de Marr (1982) y Biederman (1987), en el sentido de que el reconocimiento visual requiere la identificación de las partes del objeto y sus relaciones espaciales.

A partir de esta experiencia, se genera el interés de investigar el proceso que sigue un invidente que utiliza un dispositivo mecatrónico en el reconocimiento de patrones visuales. De la misma forma, se abre la posibilidad de identificar invariantes entre los invidentes que usan el bastón y los invidentes que utilizan el dispositivo en tareas de reconocimiento de patrones en un contexto real y en un mapa háptico.

\section{Experiencias en la construcción de mapas cognitivos como modelos mentales espaciales}

Muchas investigaciones en mapas cognitivos humanos se focalizan en la visión como órgano que transmite la información importante acerca de la disposición espacial del ambiente cercano o lejano, así como los detalles contenidos en mapas y fotografías aéreas. Debe anotarse que existen algunas personas ciegas que son capaces de navegar y formar mapas cognitivos del ambiente usando la información restante (auditiva, olfativa, táctil y propioceptiva) que está disponible para ellos (Peruch et al., 2000).
Thomsom (1983) mostró que los humanos con los ojos vendados pueden alcanzar un objetivo, habiéndolo visto previamente a una distancia considerable. Rieser (1989) y Loomis et al. (1993) han investigado acerca de la habilidad de las personas videntes e invidentes para imaginar la ubicación de un lugar conocido e indicar su localización en la oscuridad. En esta experiencia se vio que la precisión variaba considerablemente, dependiendo de la familiaridad de los participantes con el área de prueba, lo cual indica que en ambientes no familiares y con direcciones que no son bien conocidas, pueden ocurrir grandes errores en términos del recorrido o en la descripción verbal o representación del lugar transitado. En consecuencia, la longitud de un paso, derivada de las señales propioceptivas o la salida de señales motoras, así como las señales vestibulares, pueden contribuir a la actualización de la representación mental de localización en el espacio de los sujetos, permitiendo la integración del camino (Mittelstaedt y Glasauer, 1991; Glasauer et al., 1994).

En la construcción de modelos mentales espaciales, los invidentes tempranos (personas que han perdido su visión antes de los tres años de edad) normalmente actúan almacenando información visual espacial de forma pasiva, pero tienen problemas con los procesos que requieren integración y transformación activa (Cornoldi y Vecchi, 2000). Sin embargo, otros estudios han mostrado que los invidentes congénitos realizan muchas tareas de imaginería e incluso tareas que requieren procesos de imaginería mental activa con ligera desventaja respecto de los videntes (Kaski, 2002). 
Klatzky et al. (1995) afirman que existen pequeñas diferencias en la realización de tareas espaciales en videntes e invidentes, y los experimentos de Landau et al. (1984) muestran que los niños ciegos tienen un conjunto de capacidades que parecen indicar la presencia de un sistema para el conocimiento espacial; este sistema involucraría procesos complejos como inferencias espaciales que permiten la navegación a lo largo de nuevos caminos, como lo corroboran los estudios de Kaski (2002).

Estudios realizados por Noordzij y Postma (2005) evalúan la importancia de la experiencia visual en función de la habilidad de videntes e invidentes para formar una representación espacial o mapa cognitivo, después de escuchar descripciones elaboradas del espacio. Este proceso lo realizan con el fin de determinar si la experiencia visual desempeña un rol crucial en la construcción de modelos mentales espaciales y si los invidentes muestran las mismas preferencias (nivel de realización de las tareas) que los videntes en el procesamiento del tipo de perspectiva empleado en la descripción espacial. Los resultados muestran que los videntes e invidentes con o sin experiencia visual forman un modelo mental espacial que incluye la codificación de la distancia entre los objetos. En la misma experiencia los participantes, luego de percibir a través del tacto un modelo a escala de un escenario que contiene objetos distribuidos en el área, nombran la misma cantidad de ubicaciones correctas de los objetos. Esto sugiere que no existen diferencias en la habilidad para formar modelos mentales entre los dos grupos, lo cual confirma que existe una evidencia creciente respecto a que la experiencia visual no es una característica esencial en el desarrollo de representaciones espaciales. Otro resultado de esta investigación muestra que las representaciones espaciales pueden ser construidas a partir de descripciones verbales complejas de configuraciones del espacio, desconocidas previamente por los invidentes y videntes, tales como objetos ubicados en una configuración circular del espacio.

Con relación a las perspectivas basadas en la ruta y el panorama del ambiente, surge la pregunta: ¿la perspectiva espacial de estas descripciones influye en el modelo mental espacial? De acuerdo con varios estudios realizados en este campo, no se han encontrado diferencias en los modelos mentales espaciales de videntes basados en la descripción de ruta o descripción de la visión panorámica del ambiente (Taylor y Tversky, 1992; Ferguson y Hegarty, 1994). Esto sugiere que la gente utiliza modelos mentales independientes del punto de vista (Zwaan y Radvansky, 1998).

Investigaciones acerca de la forma como personas invidentes codifican el espacio en tareas hápticas y de locomoción muestran que ciertas estrategias, con respecto a la preferencia del marco de referencia, difieren con las empleadas por personas videntes (Millar, 1994; Zuidhoek et al., 2004). Millar (1994) argumenta que los ciegos tienden a codificar la información espacial, especialmente de grandes espacios, en la forma de una representación local, secuencial basada en rutas, mientras que los videntes codifican información espacial en la forma de una representación global basada en el mundo externo. Cuando los invidentes leen o escuchan 
acerca de una descripción del espacio, seleccionan la manera de construir un modelo mental espacial en forma de una representación secuencial. Las descripciones de ruta les dan información local, organizada secuencialmente, mientras que las descripciones del ambiente les dan información global, organizada jerárquicamente. Por tanto, si los ciegos construyen un modelo mental espacial en la forma de un conjunto de instrucciones procedimentales, en lugar de una representación más global, entonces la descripción de ruta puede facilitar una mejor construcción del modelo mental espacial que la descripción del ambiente (Noordzij y Postma, 2005). Los estudios realizados por Noordzij y Postma (2005) con videntes e invidentes muestran que los invidentes construyen modelos espaciales menos efectivos que los videntes en condiciones de conocimiento basado en el panorama del ambiente. En contraste, los invidentes construyen modelos mentales espaciales más efectivos que los videntes en condiciones de conocimiento basado en la ruta. Esto corrobora los planteamientos de Millar (1994) y Thinus-Blanc y Gaunet (1997), quienes argumentan que los ciegos realizan mejores representaciones y generan estrategias espaciales más eficientes, basados en información local y de ruta. Con estos planteamientos se prevé el estudio del proceso de razonamiento seguido por los invidentes para determinar las estrategias de representación espacial que les permitirían construir sus modelos mentales. Esta posibilidad permite dar una mirada a los procesos perceptivos del invidente en el reconocimiento del espacio.
Con relación a la forma en que los invidentes comprenden y comunican el lenguaje espacial, Loomis et al. (2002) mostraron que los invidentes actualizan el espacio en iguales condiciones que los videntes con base en lenguaje espacial. De la misma forma, Brambring (1982), indagando a videntes e invidentes acerca de dar información relevante de la descripción del espacio con respecto a ciertas rutas, observó que las personas videntes describen el espacio orientados en el ambiente, mientras que los invidentes lo hacen con descripciones relacionadas con su propia posición. Este patrón de comunicación del lenguaje espacial se ajusta con los resultados hallados por Noordzij y Postma (2005), según los cuales los videntes hacen descripciones basados en la globalidad, mientras que los invidentes las realizan basados en parámetros locales. Una incógnita que habría que estudiar es la forma como los invidentes comprenden las descripciones espaciales y cómo utilizan este conocimiento para buscar su propio camino.

Este objeto de estudio permitiría comparar la descripción verbal de los objetos en el ambiente, representado en un mapa táctil, con el proceso de búsqueda de los objetos representados en un escenario real, en función de identificar los procesos seguidos por la persona invidente en la percepción del espacio y en la retención o recuperación de información en la memoria. De otro lado, la descripción verbal del ambiente por parte del invidente antes de iniciar la búsqueda en el entorno, nos podría llevar a indagar si existen elementos comunes entre la forma como los sujetos 
se imaginan el ambiente y la forma como lo perciben.

Jacobson (1998) ilustra tres estudios que exploran la habilidad de personas invidentes para construir mapas cognitivos. El primer estudio prueba que los mapas táctiles son una poderosa herramienta para la orientación y aprendizaje geográfico (Ungar et al., 1997). La investigación se realiza con tres personas invidentes que viven en un campus universitario. Para realizar este estudio, los participantes, primero, externalizan su conocimiento del mapa cognitivo del campus por medio de la construcción de bocetos que indicaban su nivel básico de conocimiento del entorno (Blades, 1990). Con este método el invidente puede discernir la estructura, orden y contenido de su propio conocimiento. Posteriormente, los sujetos estudian y completan sus propios bosquejos de mapas en una tableta de líneas táctiles que contenía lo que ellos habían dibujado. Una vez ellos finalizan sus mapas, se les da un mapa táctil del campus para que exploren y hagan preguntas. Después de una semana, los participantes realizan otro boceto del campus. Los resultados de esta experiencia muestran que los mapas dibujados antes de la experiencia con el mapa táctil muestran un orden y conceptualizaban el campus de una manera holística y coherente. Los mapas dibujados después de la experiencia con el mapa táctil mejoran en detalles, adicionando algunos elementos derivados del mapa táctil y corrigiendo algunos errores.

Las conclusiones de este estudio indican que la elaboración de bocetos de mapas, pueden proveer una herramienta útil para la documentación del desarro- llo de mapas cognitivos de una persona y el uso de mapas táctiles proporcionan una visión panorámica del entorno. Con estos dispositivos, los invidentes crearon representaciones globales del espacio. Un resultado importante de este estudio es que los ciegos son capaces de producir mapas bien ordenados y con bastante precisión a partir de sus ambientes familiares. Con esto podemos pensar que un aprendizaje en adquisición de conocimiento del ambiente en el cual se desenvuelve el ciego es un elemento importante para que esta persona pueda precisar su representación espacial y pueda desenvolverse en su entorno. Este entrenamiento tendría la función de convertir el ambiente en un entorno familiar para los invidentes.

Un segundo estudio utiliza una estrategia de multianálisis y multitarea para investigar el conocimiento geográfico y el efecto de la representación de un mapa táctil de tres invidentes que viven en una región. Los invidentes estudian durante cinco minutos un mapa táctil que indica la posición y la frontera de diez ciudades ubicadas en el área donde ellos viven. Los participantes emprendieron tres tareas: la primera tarea consistía en completar una prueba de respuesta de una señal espacial. Los sujetos debían indicar en un tablero táctil la localización de las diez ciudades presentadas en el mapa táctil, ayudados por una señal espacial que consistía en conocer la ubicación y la frontera de dos ciudades. Los resultados muestran que los sujetos ubicaron las ciudades con precisión. Esta apreciación soporta la idea de que las personas ciegas son capaces de desarrollar conocimiento configuracional. Los resultados de esta experiencia sugieren 
que el conocimiento de un mapa táctil junto al conocimiento del mapa cognitivo, construido a través de la experiencia, es útil en el aprendizaje.

La segunda tarea consistía en estimar las distancias relativas para una escala multidimensional. Aquí los sujetos intentaron infructuosamente estimar distancias relativas utilizando escalado multidimensional. La tercera tarea se relacionaba con completar una prueba de reconocimiento.

Es esta tarea se le presentaron los tres mapas de forma aleatoria a los sujetos. Uno de los mapas tenía la orientación correcta, los otros fueron rotados en diferente ángulo. Los resultados mostraron que todos los sujetos identificaron el mapa con la orientación correcta.

Un tercer estudio se relacionaba con la modelación del ambiente utilizando señales auditivas. En la experiencia participaron ocho sujetos divididos en dos grupos. Todos los sujetos fueron movilizados en su entorno familiar utilizando técnicas del bastón antes de emprender la travesía de una ruta donde debían negociar obstáculos que incluían entradas y cruces de calles principales. Uno de los grupos aprendía el recorrido de manera tradicional con ayuda de un instructor en movilidad. El otro grupo recibía las mismas instrucciones que el primero, pero éste podía conocer la ruta y el área ayudado por un dispositivo audiotáctil que le daba señales auditivas del ambiente a medida que caminaba. Estas señales incluían iconos auditivos (earconos), como el ruido del tráfico, el ruido de cruce de peatones, el sonido de aviso de un cruce, etc. (Blattner et al., 1989). Cada sujeto completaba un conjunto de valoraciones cualitativas y cuantitativas, las cuales incluían descripciones verbales y bocetos de la ruta. Unas horas después de finalizar el recorrido, se le presentó a los sujetos una tablilla de dibujo táctil para que ellos describieran y dibujaran la ruta lo más detallada posible. Los sujetos podían indicar en una regla la longitud total de la ruta y la distancia a los mojones claves. Los dos grupos completaron la trayectoria de la ruta sin ayuda y fueron capaces de describir verbalmente la ruta. Los mapas dibujados por el grupo que utilizó el dispositivo fueron mejores que los del otro grupo. Sin embargo, la distribución espacial de los lugares dibujados por los dos grupos mostró un alto grado de completitud y precisión, evidenciándose mayor precisión en el grupo del dispositivo, sin ser significativa la diferencia. Las conclusiones del trabajo indican que los invidentes tienen una representación interna del espacio precisa, comparable con los videntes, aunque las representaciones de la persona ciega son cualitativa y cuantitativamente diferentes.

Los anteriores estudios demuestran claramente la necesidad de aproximarse a multitareas para analizar la habilidad de mapeo cognitivo de invidentes. En este aspecto la evolución cognitiva del invidente se ve reflejada en el aprendizaje de estrategias espaciales que le facilitan su desenvolvimiento en el entorno. Esta aproximación nos permite pensar que la investigación en mapeo cognitivo es una poderosa herramienta para diseñar estrategias de movilidad, en función de orientar procesos de aprendizaje en discapacitados visuales. 


\section{Conclusiones}

Este documento reporta una serie de antecedentes de investigación realizada con personas videntes e invidentes y nos ubica en un estado de exploración de nuevas alternativas de percepción sensorial que ayuden a personas con discapacidad visual a resolver problemas espaciales. El mapeo cognitivo, considerado un proceso de razonamiento espacial, es una estrategia poderosa que suministra información espacial, tanto a videntes como a invidentes, para su movilidad. En esta dimensión la investigación en la formación de imágenes cognitivas contribuye considerablemente al aprendizaje de la percepción del ambiente.

El interés particular por explorar la configuración que realiza el invidente del ambiente, utilizando dispositivos táctiles que transmiten información a través de órganos sensoriales paralelos como la piel, es un objetivo que se in-

\section{Bibliografía}

Aitken, S. C.; Cutter, S. L.; Foote, K. E. y Sell, J. L. (1989). Environmental perception and behavioral geography. En: Wilmott, C. y Gaille, G. (eds.). Geography in America. London: Merrill; pp. 218-238.

Allen, G. (1981). A developmental perspective on the effects of "subdividing" macrospatial experience. Journal of Experimental Psychology: Human Learning and Memory, 7, 120-132.

Allen, G.; Siegel, A. W. y Rosinski, R. R. (1978). The role of perceptual context instructuring spatial knowledge. Journal of Experimental Psychology: Human Learning and Memory, 4, 617-630. corpora al desarrollo de la investigación acerca de cómo las personas invidentes comprenden el espacio. Esto podría generar conocimiento sobre el contenido, la forma y la localización de la información espacial, para que sea disponible, tanto a los peatones invidentes como a los docentes que orientan procesos de aprendizaje en movilidad, en función de utilizar estrategias más efectivas relacionadas con el desarrollo de habilidades de orientación y búsqueda del camino. Una meta planteada en el proceso investigativo toma este conocimiento para buscar alternativas pedagógicas que mejoren el aprendizaje de invidentes en función de desenvolverse en el mundo y mejorar sus alternativas de vida. Esto es razonable con la búsqueda de estrategias para que estas personas puedan ser entrenadas en la representación de su entorno. $\boldsymbol{\Delta}$

Andrews, S. K. (1983). Spatial cognition through tactual maps. En: Wiedel, J. (ed). Proceedings of the First International Symposium on Maps and Graphics for the Visually Handicapped. Washington D. C.: Association of American Geographers, pp. 30-39.

Arno, P.; Vanlierde, A.; Streel, E.; WanedDefalque, N. C.; Sanabria-Bohórquez, S. y Veraart, C. (2001). Auditory substitution of vision: pattern recognition by the blind. Applied Cognitive Psychology, 15, 509-519.

Atkins, J. E.; Fiser, J. y Jacobs, R. A. (2001). Experience-dependent visual cue integration based on consistencies 
between visual and haptic percepts. Vision Research, 41, 449-461.

Biederman, I. (1987). Recognition by components: a theory of human image understanding. Psychological Review, 94, 115-147.

Biel, A. (1982). Children's spatial representation of their neighborhood: A step towards a general spatial competence. Journal of Environmental Psychology, 2, 193-200.

Bigelow, A. (1991). Spatial mapping of familiar locations in blind children. Journal of Visual Impairment and Blindness, 85, 113-117.

Blades, M. (1990). The reliability of date collected from sketch maps. Journal of Environmental Psychology, 10, 327-340.

Blades, M.; Lippa, Y.; Golledge, R. G.; Jacobson, R. D. y Kitchin, R. M. (2002). The effect of spatial tasks on visually impaired peoples' wayfinding abilities. Journal of Visual Impairment and Blindness, 96 (6), 407-420.

Blattner, M. M. y Sumikaua, D. A. (1989). Earcons and icons; their structure and common design principles. HumanComputer Interaction 4, 11-44.

Brambring, M. (1982). Language and geographic orientation for the blind. En: Jarvella, R. J. y Klein, W. (eds.). Speech, place, and action. Nueva York: Wiley, pp. 203-218.

Brewster, S. y Brown, L. M. (2004). Tactons: Structured Tactile Messages for NonVisual Information Display. Documento de la 5th Australasian User Interface Conference (AUIC2004). Dunedin: Australia.

Cadwallader, M. T. (1979). Problems in cognitive distance and their implications to cognitive mapping. Environment and Behavior, 11, 559-576.
Cornoldi, C. y Vecchi, T. (2000). Mental imagery in blind people: The role of passive and active visuospatial processes. En: Heller, M. (ed.). Touch, representation, and blindness. Oxford: Oxford University Press, pp. 143-181.

Downs, R. M. y Stea, D. (1973). Cognitive Maps and Spatial Behavior: Process and Products. En: Image and Environments. Chicago: Aldine Publishing Company, pp. 8-26.

Evans, G. W. y Pezdek, K. (1980). Cognitive mapping: Knowledge of real-world distance and location information. Journal of Experimental Psychology: Human Learning and Memory, 6, 13-24.

Ferguson, E. L. y Hegarty, M. (1994). Properties of cognitive maps constructed from texts. Memory y Cognition, 22, $455-473$

Garling, T.; Book, A. y Lindberg, E. (1984). Cognitive mapping of large scale environments: the interrelationship of action plans, acquisition and orientation. Environment and Behaviour $16,3-34$.

Glasauer, S.; Amorim, M. A.; Vitte, E. y Berthoz, A. (1994). Goal-directed linear locomotion in normal and labyrinthine-defective subjects. Experimental Brain Research, 98, 323-335.

Golledge, R. G. (1977). Environmental cues, cognitive mapping and spatial behaviour. En: Burke, D. et al. (eds.). Behavior-Environment research methods. Madison, WS: Institute for Environmental Studies, University of Wisconsin, pp. 35-46. . (1993). Geography and the disabled: a survey with special reference to vision impaired and blind populations. Transactions of the 
Institute of British Geographers, 18, 63-85.

(1999). Human wayfinding and cognitive maps. En: Golledge, R. (ed.). Wayfinding behaviour. Baltimore, MA: Johns Hopkins University Press, pp. 5-45.

Golledge, R. G. y Stimpson, R. J. (1997). Spatial behaviour: a geographic perspective. Nueva York: The Guilford Press.

Hart, R. A. y Moore, G. T. (1973). The development of spatial cognition: a review. En: Downs, R.; Stea, D.; ClarkCarter, D. D.; Heyes, A. D. y Howarth, C. I. (eds.). (1986). Image and Environment: cognitive mapping and spatial behaviour. Chicago: Aldine, pp. 246-288.

Hart, R. A. y Conn, M. K. (1991). Developmental perspectives on decision making and action in environments. En: Garling, T. y Evans, G. W. (eds.). Environment, Cognition and Action: an integrated approach. Nueva York: Plenum Press, pp. 277-294.

Hardwick, D. A.; McIntyre, C. W., y Pick, H. L. (1976). The content and manipulation of cognitive maps in children and adults. Monographs of the Society for Research in Child Development, 41, 1-55.

Hunt, E. y Waller, D. (1999). Orientation and wayfinding: a review. Washington D.C.: University of Washington.

Intraub, H. (2004). Anticipatory spatial representation of $3 \mathrm{D}$ regions explored by sighted observers and a deaf-andblind-observer. Cognition, 94, 19-37.

Jacobson, R. D. (1992). Spatial cognition through tactile mapping. Swansea Geographer, 29, 79-88.

(1998). Cognitive mapping without sight: four preliminary stud- ies of spatial learning. Journal of Environmental Psichology, 18, 289-305.

(1998). Navigating maps with little or no sight: An audio-tactile approach. Proceedings of Content Visualization and Intermedia Representations. Montreal: University of Montreal.

(2002). Representing Spatial Information through Multimodal Interfaces. Proceedings of the Sixth International Conference on Information Visualisation (IV'02). IEEE: Computer Society, pp. 730-734.

Kaski, D. (2002). Revision: Is visual perception a requisite for visual imagery? Perception, 31, 717-731.

Kirasic, K. C.; Allen, G. y Siegel, A. (1984). Expression of configurational knowledge of large scale environments. Environment and Behavior, 16, 687 712.

Kitchin, R. M. (1994). Cognitive maps: what are they and why study them? Journal of Environmental Psychology, 14, 1-19.

(1995). Issues of validity and integrity in cognitive mapping research. Investigating configurational knowledge. Disertación doctoral sin publicar. Swansea: University of Wales.

Kitchin, R. M. y Jacobson, R. D. (1997). Techniques to Collect and Analyzethe Cognitive Map Knowledge of Persons with Visual Impairment or Blindness: Issues of Validity. Journal of Visual Impairment and Blindness, 91, 360376.

Kitchin, R. M.; Blades, M. y Golledge, R. G. (1997). Understanding spatial concepts at the geographic scale without 
the use of vision. Progress in Human Geography, 21, 225-242.

Klatzky, R. L.; Loomis, J. M.; Golledge, R. G.; Cicinelli, J.; Doherty, S. y Pellegrino, J. (1990). Acquisition of route and survey knowledge in the absence of vision. En: Journal of Motor Behavior, $22,19-43$.

Klatzky, R. L.; Golledge, R. G.; Loomis, J. M.; Cicinelli, J. G. y Pellegrino, J. W. (1995). Performance of blind and sighted persons on spatial tasks. Journal of Visual Impairment and Blindness, 89, 70-82.

Klatzky, R. L. y Lederman, S. J. (2003). Representing Spatial Location and Layout From Sparse Kinesthetic Contacts. Journal of Experimental Psychology: Copyright by the American Psychological Association, Inc. Human Perception and Performance, 29(2), 310-325.

Landau, B.; Spelke, E. y Gleitman, L. (1984). Spatial knowledge in a young blind child. Cognition, 16, 225-260.

Lockman, J. J.; Rieser, J. J. y Pick, H. L. (1981). Assessing blind travelers' knowledge of spatial layout. Journal of Visual Impairment and Blindness, 75, 321-326.

Loomis, J. M.; Klatzky, R. L.; Golledge, R. G.; Cicinelli, J. G.; Pellegrino, J. W. y Fry, P. A. (1993). Nonvisual navigation by blind and sighted: assessment of path integration ability. Journal of Experimental Psychology, 122, 73-91.

Loomis, J. M.; Lippa, Y.; Golledge, R. G., y Klatzky, R. L. (2002). Spatial updating of locations specified by 3D sound and spatial language. Journal of Experimental Psychology: Learning, Memory and Cognition, 28, 335-345.

Marr, D. (1982). Vision: A computational investigation into the human represen- tation and processing of visual information. San Francisco, CA: Freeman.

Millar, S. (1994). Understanding and representing space: Theory and evidence from studies with blind and sighted children. Oxford-England: Clarendon Press-Oxford University Press.

(1997). La comprensión y la representación del espacio: Teoría y evidencia a partir de estudios con niños ciegos y videntes. Traducido por Soledad Ballesteros. Madrid: Servicios Sociales ONCE.

Mittelstaedt, H. y Glasauer, S. (1991). Idiothetic navigation in gerbils and humans. Zoological Journal Physiology, 95, 427-435.

Montagu, A. (1971). Touching: The Human Significance of the Skin. Nueva York: Columbia University Press.

Montello, D. R. (1991). The measurement of cognitive distance: Methods and construct validity. Journal of Environmental Psychology, 11, 101-122.

Noordzij, M. L. y Postma, A. (2005). Categorical and metric distance information in mental representations derived from route and survey descriptions. Psychological Research, 69, 221-232.

O’Regan, J. K. (1992). Solving the real mysteries of visual perception: The world as an outside memory. Canadian Journal of Psychology, 46, 461-488.

Passini, R. y Proulx, G. (1988). Wayfinding without vision. An experiment with congenitally totally blind people. Environment and Behaviour, 20, 227-252.

Passini, R.; Proulx, G. y Rainville, C. (1990). The spatio-cognitive abilities of the visually impaired population. Environment and Behaviour, 22, 91-118. 
Peruch, P.; Gaunet, F.; Thinus-Blanc, C. y Loomis, J. (2000). Understanding and learning virtual spaces. En: Kitchin, R. y Freundschuh, S. (eds.). Cognitive mapping: past, present and future. London: Routledge.

Revesz, G. (1950). Psychology and art of the blind. Nueva York: Longmans.

Rieser, J. J. (1989). Access to knowledge of spatial structure at novel points of observation. Journal Experimental Psychology Learning Memory Cognition, 15, 1157-1165.

Robinson, A. H.; Sale, R. y Morrison, N. J. (1978). Elements of Cartography. $4 .^{\mathrm{a}}$ ed. Nueva York: John Wiley and Son.

Rosa, A. y Ochaíta, E. (1993). Introducción. ¿Puede hablarse de una psicología de la ceguera? En: Rosa, A. y Ochaíta, E. (comps.). Psicología de la ceguera. Madrid: Alianza, pp. 1-18.

Taylor, H. A., y Tversky, B. (1992). Spatial mental models derived from survey and route descriptions. Journal of Memory and Language, 31, 261-292.

Thinus-Blanc, C. y Gaunet, F. (1997). Representation of space in blind per-sons: Vision as a spatial sense? Psychological Bulletin, 121, 20-42.

Thomdyke, P. W. y Hayes-Roth, B. (1982). Differences in spatial knowledge acquired from maps and navigation. Cognitive Psychology, 14, 560-589.
Thomson, J. (1983). Is continuous visual monitoring necessary in visually guided locomotion? Journal Experimental Psychology, 9, 427-443.

Ungar, S.; Blades, M. y Spencer, C. (1997). Teaching visually impaired to make distance judgements from a tactile map. Journal of Visual Impairment and Blindness, 91, 163-174.

Walter, I. I. y Robinson, A. (1983). Cartographic Innovations: International Handbook of Mapping Terms to IWO. Nueva York: Map Collectors Publications Ltd.

Warren, D. H. y Kocon, J. (1974). Factors in the successful mobility of the blind: a review. American Foundation for the Blind Research Bulletin, 28, 191-218.

Zuidhoek, S.; Kappers, A. M. L.; Noordzij, M. L.; Van der Lubbe, R. H. J. y Postma, A. (2004). Frames of reference in a haptic parallelity task: Temporal dynamics and the possible role of vision. En: Ballesteros, S. y Heller, M. A. (eds.). Touch, blindness and neuroscience. Madrid: UNED Press; pp. 155-164.

Zwaan, R. A., y Radvansky, G. A. (1998). Situation models in language comprehension and memory. Psychological Bulletin, 13, 162-85. 